\title{
The Dilogarithm Function of a Real Argument
}

\section{By Robert Morris}

Abstract. This paper is a user's guide to the dilogarithm function

$$
L i_{2}(z)=-\int_{0}^{z} \frac{\log (1-z)}{z} d z
$$

of a real argument. It is intended for those who are primarily interested in the values of the dilogarithm rather than in its functional relationships.

The paper is deliberately written in the style of the book Computer Approximations by Hart, Cheney et al. [1] by

$$
L i_{2}(z)=-\int_{0}^{z} \frac{\log (1-z)}{z} d z
$$

The function is real-valued for real values of $z \leqslant 1$ and has a logarithmic branch point at $z=1$. It is usual to assign a branch cut along the real line from 1 to $\infty$ and to assign the imaginary part $-i \pi \log (x)$ to $L i_{2}(x)$ for real values of $x>1$. In what follows, we deal only with the real part of the function $L i_{2}(x)$ for real arguments $x$.

$L i_{2}$ is asymptotic to $\pi^{2} / 3-1 / 2 \log ^{2}(x)$ for large $x$ and to $-\pi^{2} / 6-1 / 2 \log ^{2}(-x)$ for large negative $x$.

$L i_{2}$ has a maximum at $x=2$ and the value there is $\pi^{2} / 4$.

$L i_{2}$ has a zero at the origin and at $x=12.5951703698450161286398965 \ldots$ [4] . $L i_{2}$ has infinite slope at $x=1$.

b. Fundamental Identities.

1. Expansions.

$$
L i_{2}(x)=\frac{x}{1^{2}}+\frac{x^{2}}{2^{2}}+\frac{x^{3}}{3^{2}}+\frac{x^{4}}{4^{2}}+\cdots, \quad-1 \leqslant x \leqslant 1,
$$

$$
L i_{2}\left(e^{-z}\right)=\frac{\pi^{2}}{6}+z\left(\log (z)-1-\frac{z}{4}+\frac{B_{2} z^{2}}{2 \cdot 3 \cdot 2 !}-\frac{B_{4} z^{4}}{4 \cdot 5 \cdot 4 !}+\cdots\right),
$$

where $B_{n}$ are the Bernoulli numbers $B_{2}=1 / 6, B_{4}=-1 / 30$, etc., with the notation of [2]. A comparable expression for $L i_{2}\left(-e^{-z}\right)$ can be derived by the use of Eq. (11).

2. Functional Relations. There are a number of functional relationships, all stated and derived in [1], which serve to reduce the computation of the dilogarithm to the interval $0 \leqslant x \leqslant 1 / 2$.

Received May 22, 1978.

AMS (MOS) subject classifications (1970). Primary 65D20, 33A10, 41 A50.

(C) 1979 American Mathematical Society 0025-5718/79/0000-0072/\$03.50 


$$
L i_{2}(x)=\frac{\pi^{2}}{6}-\log (x) \log (1-x)-L i_{2}(1-x), \quad \frac{1}{2}<x<1,
$$

$$
L i_{2}(x)=\frac{\pi^{2}}{6}-\log (x) \log (x-1)-\frac{1}{2} \log (x)+L i_{2}\left(1-\frac{1}{x}\right), \quad 1<x \leqslant 2,
$$

$$
\begin{gathered}
L i_{2}(x)=\frac{\pi^{2}}{3}-\frac{1}{2} \log ^{2}(x)-L i_{2}\left(\frac{1}{x}\right), \quad 2<x<\infty, \\
L i_{2}(x)=-\frac{1}{2} \log ^{2}(1-x)-L i_{2}\left(\frac{x}{x-1}\right), \quad-1 \leqslant x<0, \\
L i_{2}(x)=-\frac{\pi^{2}}{6}-\frac{1}{2} \log (1-x)[2 \cdot \log (-x)-\log (1-x)]+L i_{2}\left(\frac{1}{1-x}\right), \\
-\infty<x<-1 .
\end{gathered}
$$

The intervals stated are those which provide for reduction to the range $[1 / 2,1]$, but the relationships are valid over much wider intervals.

3. Improved Convergence. It is possible to improve the $n^{2}$ rate of convergence of series (2) by well-known techniques. If the expression

$$
\frac{1}{n(n+1)}=\frac{1}{n}+\frac{-1}{n+1}
$$

is squared, multiplied by $x^{n}$ and the infinite sum expanded in terms of logarithms and dilogarithms, then we obtain a series with $n^{4}$ convergence

$$
L i_{2}(x)=\left(\frac{x}{x+1}\right)\left[3+\sum_{1}^{\infty} \frac{x^{n}}{n^{2}(n+1)^{2}}\right]-2 \cdot\left(\frac{x-1}{x+1}\right) \log (1-x) .
$$

The $n^{6}$ analog is

$$
\begin{aligned}
\left(1+4 x+x^{2}\right) L i_{2}(x)= & 4 x^{2} \sum_{1}^{\infty} \frac{x^{n}}{[n(n+1)(n+2)]^{2}} \\
& +4 x+\frac{23}{4} x^{2}+3\left(1-x^{2}\right) \log (1-x)
\end{aligned}
$$

and both of these series converge for $|x| \leqslant 1$.

One can go arbitrarily far in this direction, but the expressions get more complex and are of little use for machine computation. On the other hand, they certainly are convenient for hand computation. The $n^{6}$ version of this procedure is used in [3] but in that case it only succeeds in getting wrong answers faster, as the resulting expression has enormous internal cancellation for arguments near 0 .

c. Error Propagation. For values of $x<0$, both the absolute derivative $d f / d x$ and the relative derivative $x / f \cdot d f / d x$ are less than 1 in magnitude and so in this interval both relative errors and absolute errors in the argument are damped.

In the interval $0 \leqslant x \leqslant 2$, both of the derivatives rise from 1 at the origin, become infinite at $x=1$, and then decrease to zero at $x=2$. Therefore, both relative and absolute accuracy are completely lost in the neighborhood of the point $x=1$. The absolute error is amplified in the interval $0 \leqslant x \leqslant 1.28 \ldots$, and the relative error in 
the interval $0 \leqslant x \leqslant 1.13 \ldots$; they are damped otherwise.

In the interval $x>2$, the derivative goes from zero towards its asymptote of $-\log (x) / x$ and is less than 1 throughout the interval. Therefore, absolute errors are damped for $x>2$. The same is true for relative error except that near the zero at $x=$ $12.595 \ldots$,.., all relative accuracy is lost.

d. Design of Subroutines. Because the function has value 0 and slope 1 at the origin, it is reasonable to look for relative accuracy near the origin. For this reason, the error curves for $\mathrm{Li}_{2}(x) / x$ were leveled.

The use of Eqs. (4) through (8) to bring arguments into the range $[0,1 / 2]$ causes little loss of precision. Neither (4) nor (5) is valid for $x=1$, which must be treated separately.

The series (3) can be used near $x=1$ and no problems arise, but internal cancellation will cause loss of significance if the series is used for arguments near zero. For arguments larger than about 2, it is more practical to use Eq. (6). The convergence of series (3) is quite good, but little is gained by its use because of the two logarithms that are required.

Equations (9) and (10) have improved convergence, but are not very useful for machine computation. There is some error amplification with either series because of partial cancellation, but far more important is the complete loss of accuracy due to internal cancellation for small values of $x$. In the algorithm published in [3], Eq. (10) was used and led to the loss of two decimal places of accuracy for arguments near .01 . The problem would be solved if a routine were used which returned accurate values of $\log (1+x)$ as a function of $x$.

It does not seem necessary or practical to provide any remedy for the loss of relative significance near the zero at $x=12.595 \ldots$, since normally a standard of absolute accuracy is what is wanted in this range; and, in any case, little improvement could be obtained without a separate entry point.

No alarm exits are required and no overflow can occur. Some loss of precision will be experienced if the polynomials in the tables are evaluated in the normal way because the coefficients are not a decreasing sequence. The loss is not great, and full accuracy can be obtained by converting them to continued fraction form by the methods described in [5].

e. Checking. Consistency checks can be derived from the relationship

$$
L i_{2}(x)+L i_{2}(-x)=\frac{1}{2} L i_{2}\left(x^{2}\right)
$$

and the series (3) can be used for arguments near 1. There are also some special values for which the value of $L i_{2}$ can be expressed in simple terms. These are all derived in [1].

$$
\begin{gathered}
L i_{2}(1)=\frac{\pi^{2}}{6}, \quad L i_{2}(-1)=\frac{-\pi^{2}}{12}, \quad L i_{2}\left(\frac{1}{2}\right)=\frac{\pi^{2}}{12}-\frac{1}{2} \log ^{2}(2), \\
L i_{2}(2)=\frac{\pi^{2}}{4}, \quad L i_{2}(\phi)=\frac{\pi^{2}}{10}-\log ^{2}(\phi)
\end{gathered}
$$


where $\phi=(\sqrt{5}-1) / 2$. From this last equation, additional special values can be obtained by the use of (4)-(8) for the arguments $-\phi, 1+\phi, 1-\phi,-1-\phi, 2+\phi$.

In addition, a host of two-term expressions can be derived from the relationships given in [1], for example,

$$
6 L i_{2}(3)-3 L i_{2}(-3)=2 \pi^{2}
$$

f. Constants. The following constants are needed for constructing or testing the dilogarithm.

$$
\begin{aligned}
\frac{\pi^{2}}{6} & =1.644934066848226436472415166646, \\
\phi & =0.618033988749894848204586834366, \\
\log 2 & =0.693147180559945309417232121458
\end{aligned}
$$

Tables of values of $L i_{2}$ are given to 30D in Appendix B for testing purposes.

g. A Priori Calculation. Series (2) and relations (4) through (8) are entirely satisfactory for a priori calculation, and the values in Appendix B were calculated in this way.

h. Index Tables. The code name for the dilogarithm is DILOG. Coefficients for rational approximations to $L i_{2}(x)$ are given in Appendix A. The coefficients are given to enough decimal places that each of them ends with some meaningless digits. $\mathrm{Pn}$ is the coefficient of $x^{n}$ in the numerator and Qn is the coefficient of $x^{n}$ is the denominator. The error criterion for these approximations is relative.

Index for the Dilogarithm Function

$\begin{array}{rrrrr}\frac{L i_{2}(x)}{x} \approx & \frac{P(x)}{Q(x)} & & & \\ \text { Range } & \text { Precision } & \text { N } & \text { M } & \text { Index } \\ & & & & \\ {[0,1 / 2]} & 2.5 & 0 & 1 & 0001 \\ & 3.9 & 1 & 1 & 0002 \\ 5.3 & 1 & 2 & 0003 \\ 6.7 & 2 & 2 & 0004 \\ 8.1 & 2 & 3 & 0005 \\ 9.4 & 3 & 3 & 0006 \\ 10.8 & 3 & 4 & 0007 \\ 12.1 & 4 & 4 & 0008 \\ 13.5 & 4 & 5 & 0009 \\ 14.9 & 5 & 5 & 0010 \\ 16.2 & 5 & 6 & 0011 \\ 17.6 & 6 & 6 & 0012 \\ 19.0 & 6 & 7 & 0013 \\ 20.3 & 7 & 7 & 0014 \\ 21.7 & 7 & 8 & 0015 \\ 23.1 & 8 & 8 & 0016 \\ 24.5 & 8 & 9 & 0017\end{array}$


DILOG 0001

$$
\begin{aligned}
& \text { P00 -.35218 119 el } \\
& \text { Q00 -.35315 131 el } \\
& \text { Q01 }+.10000000 \mathrm{el}
\end{aligned}
$$

\section{DILOG 0002}

$$
\begin{aligned}
& \mathrm{P} 00-.200713706 \mathrm{el} \\
& \mathrm{P} 01+.505175398 \mathrm{e} 0 \\
& \mathrm{Q} 00-.200688475 \mathrm{el} \\
& \mathrm{Q} 01+.100000000 \mathrm{el}
\end{aligned}
$$

\section{DILOG 0003}

$$
\begin{aligned}
& \text { P00 }+.1120262486 \mathrm{e} 2 \\
& \text { P01 }-.6099487077 \mathrm{el} \\
& \text { Q00 }+.1120267809 \mathrm{e} 2 \\
& \text { Q01 }-.8902698834 \mathrm{el} \\
& \text { Q02 }+.1000000000 \mathrm{el}
\end{aligned}
$$

\section{DILOG 0004}

$$
\begin{aligned}
& \mathrm{P} 00+.472557235287 \mathrm{el} \\
& \mathrm{P} 01-.369332589850 \mathrm{el} \\
& \mathrm{P} 02+.307338454738 \mathrm{e} 0 \\
& \mathrm{Q} 00+.472557132136 \mathrm{el} \\
& \mathrm{Q} 01-.487464171799 \mathrm{el} \\
& \mathrm{Q} 02+.100000000000 \mathrm{el}
\end{aligned}
$$

\section{DILOG 0005}

$$
\begin{aligned}
& \mathrm{P} 00-.35678644614688 \mathrm{e} 2 \\
& \mathrm{P} 01+.38344985841892 \mathrm{e} 2 \\
& \mathrm{P} 02-.82200395456182 \mathrm{e} 1 \\
& \mathrm{Q} 00-.35678644921010 \mathrm{e} 2 \\
& \mathrm{Q} 01+.47264679701406 \mathrm{e} 2 \\
& \mathrm{Q} 02-.16072491087932 \mathrm{e} 2 \\
& \mathrm{Q} 03+.10000000000000 \mathrm{e} 1
\end{aligned}
$$

\section{DILOG 0006}

$$
\begin{aligned}
& \mathrm{P} 00-.120942733256777 \mathrm{e} 2 \\
& \mathrm{P} 01+.159731928684940 \mathrm{e} 2 \\
& \mathrm{P} 02-.517104330177459 \mathrm{e} 1 \\
& \mathrm{P} 03+.210905759373841 \mathrm{e} 0 \\
& \mathrm{Q} 00-.120942733209355 \mathrm{e} 2 \\
& \mathrm{Q} 01+.189967605113668 \mathrm{e} 2 \\
& \mathrm{Q} 02-.857640863860377 \mathrm{el} \\
& \mathrm{Q} 03+.100000000000000 \mathrm{el}
\end{aligned}
$$

\section{DILOG 0007}

$$
\begin{aligned}
& \text { P00 }+.114570792185155 \mathrm{e} 3 \\
& \text { P01 }-.184536098711386 \mathrm{e} 3 \\
& \text { P02 }+.853711000743272 \mathrm{e} 2 \\
& \text { P03 }-.100469594382901 \mathrm{e} 2 \\
& \text { Q00 }+.114570792186959 \mathrm{e} 3 \\
& \text { Q01 }-.213178797097653 \mathrm{e} 3 \\
& \text { Q02 }+.125935722061229 \mathrm{e} 3 \\
& \text { Q03 }-.250051599362086 \mathrm{e} 2 \\
& \text { Q04 } 2.100000000000000 \mathrm{el}
\end{aligned}
$$

\section{DILOG 0008}

$$
\begin{aligned}
& \mathrm{P} 00+.32595307208543165159 \mathrm{e} 2 \\
& \mathrm{P} 01-.60686623785288001793 \mathrm{e} 2 \\
& \mathrm{P} 02+.35267277576934034438 \mathrm{e} 2 \\
& \mathrm{P} 03-.64953308147263288026 \mathrm{el} \\
& \mathrm{P} 04+.15545344775257884132 \mathrm{e} 0 \\
& \mathrm{Q} 00+.32595307208519873078 \mathrm{e} 2 \\
& \mathrm{Q} 01-.68835450581866112910 \mathrm{e} 2 \\
& \mathrm{Q} 02+.48854439198733497920 \mathrm{e} 2 \\
& \mathrm{Q} 03-.13097760478646637907 \mathrm{e} 2 \\
& \mathrm{Q} 04+.10000000000000000000 \mathrm{el}
\end{aligned}
$$

\section{DILOG 0009}

$$
\begin{aligned}
& \text { P00 - } .37051650371035162272 \text { e3 } \\
& \mathrm{P} 01+.79633014794291654315 \mathrm{e} 3 \\
& \text { P02 -.5746307648 } 1320703737 \text { e3 } \\
& \mathrm{P} 03+.15564695464190362861 \mathrm{e} 3 \\
& \text { P04 -.116635270060940 } 89867 \text { e2 } \\
& \text { Q00 -.37051 } 650371036239104 \text { e3 } \\
& \mathrm{Q} 01+.88895927387366153621 \mathrm{e} 3 \\
& \text { Q02 - .75570 } 208302525665596 \text { e3 } \\
& \mathrm{Q} 03+.26895650722565385462 \mathrm{e} 3 \\
& \text { Q04 - .35675 } 078974222689707 \text { e2 } \\
& \mathrm{Q} 05+.10000000000000000000 \mathrm{el}
\end{aligned}
$$

\section{DILOG 0010}

$$
\begin{aligned}
& \text { P00 - }-91023774918104907308 \text { e2 } \\
& \mathrm{P} 01+.21879457285794616534 \mathrm{e} 3 \\
& \text { P02 - .18477 } 140119136616282 \mathrm{e} 3 \\
& \mathrm{P} 03+.63948885505284588473 \mathrm{e} 2 \\
& \text { P04 -.77002 } 690131110915982 \mathrm{el} \\
& \text { P05 +.1201930729 } 9778598757 \text { e0 } \\
& \text { Q00 -.91023774918104787870 e2 } \\
& \mathrm{Q} 01+.24155051658743002759 \mathrm{e} 3 \\
& \text { Q02 -.23504 } 527756701175740 \mathrm{e3} \\
& \mathrm{Q} 03+.10156024448246398449 \text { e3 } \\
& \text { Q04 -.1843014376 } 3300095898 \text { e2 } \\
& \mathrm{Q} 05+.10000000000000000000 \mathrm{el}
\end{aligned}
$$




\section{DILOG 0011}

P00 +.12050 991261341671705797 e4 P01 - .3240760925 4539474989552 e4 $\mathrm{P} 02+.31928976938241289723624$ e 4 P03 - .13920671465266965241768 e4 $\mathrm{P} 04+.25212662919363406616773 \mathrm{e} 3$ P05 -.13120034432716341584577 e2 $\mathrm{Q} 00+.12050991261341672354682 \mathrm{e} 4$ Q01 -.35420 357069875166000880 e4 $\mathrm{Q} 02+.39445067176691511608434 \mathrm{e} 4$ Q03 - .2059952998.3831116588803 e4 Q04 +.502009622027681 16987420 e3 Q05 - .48063997258736084391455 e2 Q06 +.10000 000000000000000000 el

\section{DILOG 0012}

$\mathrm{P} 00+.26082984916294204666308$ e3 P01 - .76841 393881439059816780 e 3 $\mathrm{P} 02+.85348275353201671957160 \mathrm{e} 3$ P03 -.43992891356875187758 195 e3 $\mathrm{P} 04+.10321326943721726909276 \mathrm{e} 3$ P05 - .88086 82055 7588069558540 el $\mathrm{P} 06+.09619215083480205029510 \mathrm{e} 0$ $\mathrm{O} 00+.26082984916294204603695 \mathrm{e} 3$ $\mathrm{Q} 01-.83362140110512579870690 \mathrm{e} 3$ $\mathrm{Q} 02+.10329070094568341906278$ e4 Q03 -.62183293138199408960331 e3 $\mathrm{Q} 04+.18557220037537061281857 \mathrm{e} 3$ Q05 - .24566289705568547746 454 e2 Q06 +.10000 000000000000000000 el

\section{DILOG 0013}

$\mathrm{P} 00-.3937656825065493900869818$ e4 $\mathrm{P} 01+.1271863208120745404054796$ e5 P02 - .1591509794821768554584827 es $\mathrm{P} 03+.9655192830342635677723740 \mathrm{e} 4$ P04 - .28875 69599936624464997881 e4 P05 +.3774498030273331313491492 e3 P06 - .1444966290662060555999854 e2 Q00 - .3937656825065493901264089 e4 $\mathrm{Q} 01+.1370304628747382774077041$ es Q02 - .18903 34209507888708756612 es $\mathrm{Q} 03+.1310457120707158025763505$ e5 Q04 - .4762275177788010698293108 e4 Q05 +.8546715159145654253250347 e3 Q06 - .62157 $70486628979424942839 \mathrm{e} 2$ $\mathrm{Q} 07+.1000000000000000000000000 \mathrm{el}$

\section{DILOG 0014}

P00 - .7622868752683075556529146 e3 $\mathrm{P} 01+.2659322028106967649663031$ e4 P02 - .3665875881690951113352891 e4 $\mathrm{P} 03+.2524259912689578637099977$ e4 P04 - .90015670406189356220 23769 e3 P05 +.1541486633638432641369576 e3 P06 - .98371 85185 510472984934359 el $\mathrm{P} 07+.0790199588749903552752300 \mathrm{e} 0$ $\mathrm{Q} 00-.7622868752683075556495060 \mathrm{e} 3$ $\mathrm{Q} 01+.2849893746924044536339191$ e4 Q02 -.4293650776 725483382087482 e4 $\mathrm{Q} 03+.3328660675805869470518154 \mathrm{e} 4$ Q04 - 1402876448660074304411522 e4 Q05 +.3105486484172075590743310 e3 Q06 - .3150128765225524806446416 e2 $\mathrm{Q} 07+.1000000000000000000000000 \mathrm{el}$

\section{DILOG 0015}

$\mathrm{P} 00+.1291524527680671904833413153$ es P01 - .48707489979628478978 83707474 es $\mathrm{P} 02+.7398021817321339085128615142$ e5 P03 - .57734 445116765313018 85864690 es P04 +.24413475092656133778 64169145 es P05 -.5382475484018226420583399478 e4 $\mathrm{P} 06+.5340160722157634524303757297 \mathrm{e} 3$ P07 - .15675 76496907854205887741061 e2 $\mathrm{Q} 00+.1291524527680671904833648966$ e5 Q01 - .5193630129883015874269853176 es Q02 +.8552926624494240642249934724 e5 Q03 - .741532643634868 8375591676 737 es Q04 +.3617794839863916829152183411 es Q05 -.9814594889340097810109998 809 e4 $\mathrm{Q} 06+.1360399725084658895767875526 \mathrm{e} 4$ Q07-.7794549609017792111840945980 e2 Q08 +.1000000000 000000000000000000 el 


\title{
DILOG 0016
}

\begin{abstract}
$\mathrm{P} 00+.2263249801882465156637263082$ e4
P01 - .9123768147225415733240743276 e4

P02 +.150289706922976 1669238432998 es

P03 -.12983 35516 47249 3691814757240 es

P04 +.62678 10607037989316233309136 e4

P05 -.16596 90181 28602 5874078635080 e4

$\mathrm{P} 06+.2177688398667096125657883458$ e3

P07 -.10798 42257419338533423708485 e2

$\mathrm{P} 08+.0662537985854500873277232000 \mathrm{e} 0$

$\mathrm{Q} 00+.2263249801882465156637244265$ e4

Q01 - .96895 80597696032022384242575 e4

$\mathrm{Q} 02+.1719989364151246190055387137$ e5

Q03 -.16348 16162131003611349349316 es

Q04 +.8958820514144813267199807015 e4

Q05 - .2833211086 193356515445871 761 e4

$\mathrm{Q} 06+.4873777106496420921064865660 \mathrm{e} 3$

Q07 -.39232 99443203292023103784706 e2

Q08 +. 1000000000000000000000000000 el
\end{abstract}

\section{DILOG 0017}

P00 - .42486 0941577076578425815513413 es

$\mathrm{P} 01+.183244531779306992722635513651$ e6

P02 -.32752 6669297550162522223586638 e6

$\mathrm{P} 03+.313122440690220044970422956820$ e6

P04 -.17221 8697819825427580876931586 e6

$\mathrm{P} 05+.544281929261565482937728842213$ e5

P06 -.92731 7883956725077226221332049 e4

P07 +.723979649523300 321037522923595 e3

P08 -.16815 16155 14046 449580975569676 e2

Q00 -.42486 0941577076578425815660209 es

$\mathrm{Q} 01+.193866055318733907183294751241 \mathrm{e} 6$

Q02 - .37127 2505998599455115517656852 e6

$\mathrm{Q} 03+.387055275261533981150821382566$ e6

Q04 - .23814720065981043990 2217446162 e6

$\mathrm{Q} 05+.875889100897049125433126845284$ es

Q06 -.18567 7658098068966095883646794 es

Q07 +.205632929098181 836999766662708 e4

Q08 - .95414 1875991525514141234686148 e2

Q09 +. 100000000000000000000000000000 el 
$\mathbf{x}$

$0.02 \quad 0.020100899018693195540755412493$

$0.04 \quad 0.040407275324338307148543884616$

$0.06 \quad 0.060924842459884974299798979316$

$0.08 \quad 0.081659587698643265198479878575$

$0.10 \quad 0.102617791099391131113837369057$

0.120 .123806046327036042419159027599

$0.14 \quad 0.145231283445341062927691619326$

$0.16 \quad 0.166900793917664072947938365447$

$0.18 \quad 0.188822258086085853335161595038$

$0.20 \quad 0.211003775439704772611185096074$

$0.22 \quad 0.233453898030463794829030055518$

$0.24 \quad 0.256181667451142899587256342099$

$0.26 \quad 0.279196655856947818063632058343$

$0.28 \quad 0.302509011591760420807488203837$

$0.30 \quad 0.326129510075476069530035694175$

$0.32 \quad 0.350069610723578473188921461188$

$0.34 \quad 0.374341520808806402255075418013$

$0.36 \quad 0.398958267343333705829727779501$

$0.38 \quad 0.423933778265871398349749633474$

$0.40 \quad 0.449282974471281664464733402376$

$0.42 \quad 0.475021874533404081393528930687$

$0.44 \quad 0.501167714361562439055900777479$

$0.46 \quad 0.527739084519805883772642790164$

$0.48 \quad 0.554756088554961362938602345297$

$0.50 \quad 0.582240526465012505902656320160$

$0.52 \quad 0.610216108447686308560064263976$

$0.54 \quad 0.638708705375584146551789292715$

$0.56 \quad 0.667746644153605339596512280099$

$0.58 \quad 0.697361058374981589284339230087$

$0.60 \quad 0.727586307716333389513536296840$

$0.62 \quad 0.758460483608688037400563251928$

$0.64 \quad 0.790026024347134536378400364632$

$0.66 \quad 0.822330470644328180452391505023$

$0.68 \quad 0.855427403747997710063983628277$

$0.70 \quad 0.889377624286038738601006274807$

$0.72 \quad 0.924250653636126098873386769629$

0.740 .960126675233516956781208711469

$0.76 \quad 0.997099088305810395911743645829$

$0.78 \quad 1.035277934217013403697392723810$

$0.80 \quad 1.074794600008248359395451922854$

$0.82 \quad 1.115808450985135482650401791877$

$0.84 \quad 1.158516487507309750805725217889$

$0.86 \quad 1.203167960860418347404547420250$

$0.88 \quad 1.250087584199261871567654759981$

$0.90 \quad 1.299714723004958725171060494193$

$0.92 \quad 1.352675161043712628374329977602$

$0.94 \quad 1.409928300464020368928954559279$

$0.96 \quad 1.473125860239736728262269485409$

$0.98 \quad 1.545799712031465609713051762219$

1.001 .644934066848226436472415166646
$-.019900879015136839780342951326$

-.039606955096577741644497190472

$-.059123219862624050751326518607$

-.078454453082443202331042859707

-.0976052352 29321583841103341851

$-.116579959082834610114664573331$

$-.135382840467294849314621822263$

$-.154017928204449018083640434686$

$-.172489113350156305978567089030$

-.190800137777535619036913153766

-.208954602162683753615964017283

-.226955973423426039147913551882

$-.244807591656568191553058387318$

$-.262512676614689638281448110532$

-.280074333759582904230216972305

-.297495559925939290064512421852

-.314779248625755016545532916910

-.331928195021138439164407701793

-.348945100590698 179124418838894

-.365832577512449627990764219653

-.382593152784163303335229665162

-.399229272100266 844015098449953

-.415743303502780223139440376484

$-.432137540822291058100935672666$

-.44841 4206923646202443064405916

$-.464575456769831733127134855858$

$-.480623380316422462665913464193$

$-.496560005247992274621315234110$

-.512387299566977363848638322300

-.528107174044666536598672407090

-.543721484543247829732934686901

-.559232034217161512400276604810

-.574640575601389522398739806762

-.589948812593744763913894824356

-.6051584023 37705283974426887577

-.6202709570118635372363675 23198

$-.635288045531625617630504710184$

-.65021 119516839578868 2061825034

-.665041893091114572564697019132

$-.679781587834681091206251740538$

-.694431690699479622285071072771

-.708993577085944037747757898548

$-.723468587767829769834925149256$

$-.737858030107619249168943827238$

$-.752163179217261620372692713427$

$-.766385279067239371382567985458$

$-.780525543546761872965963982604$

-.794585157477707410460367048107

-.8085652775 84769917544493333163

-.822467033424113218236207583323 


$$
\operatorname{dilog}(x)
$$

$1.1 \quad 1.961999101305568593053176992766$

$1.2 \quad 2.129169430383959659444305569819$

$1.3 \quad 2.240887839853646066434666550977$

$1.4 \quad 2.319073036309661140597949062073$

$1.5 \quad 2.374395270272480200677499763072$

$1.6 \quad 2.413131137974625258433089529909$

$1.7 \quad 2.439354270885838956416859251849$

$1.8 \quad 2.455876458504301735012301543645$

1.92 .464723302489591317096116958476

$2.0 \quad 2.467401100272339654708622749969$

$2.1 \quad 2.465057975380808733171630566323$

$2.2 \quad 2.458586601999741851737986678759$

$2.3 \quad 2.448692515743370111823655739529$

$2.4 \quad 2.435941099045935577469203445568$

$2.5 \quad 2.420790806565933843913656593893$

$2.6 \quad 2.403617212268632402133173451324$

$2.7 \quad 2.384730761537743347168527864660$

$2.8 \quad 2.364390102328581973568210913407$

$2.9 \quad 2.342812247293540603117407553046$

$3.0 \quad 2.320180423313098396406194473703$

$3.1 \quad 2.296650206578988948078244290375$

$3.2 \quad 2.272354368691871251464108780631$

$3.3 \quad 2.247406741367447881020541916266$

$3.4 \quad 2.221905325395980123780617533989$

$3.5 \quad 2.195934811575780804252856100843$

$3.6 \quad 2.169568639783855065339789579840$

$3.7 \quad 2.142870692126057632221233634587$

$3.8 \quad 2.115896693859746744480291920201$

$3.9 \quad 2.088695379215163270851814148904$

$4.0 \quad 2.061309466777317416691441452151$

$4.1 \quad 2.033776479621663456648249864539$

$4.2 \quad 2.006129438139823827310544507153$

$4.3 \quad 1.978397447883292851120445756021$

$4.4 \quad 1.950606200385219567506438445576$

$4.5 \quad 1.922778401493970986029562731318$

$4.6 \quad 1.894934139046013164770373951243$

$4.7 \quad 1.867091199554661796866627371950$

$4.8 \quad 1.839265341871258900493067876821$

$4.9 \quad 1.811470534392159447938431229342$

$5.0 \quad 1.783719161266630627743559734722$

$5.1 \quad 1.756022202152051164244223774355$

$5.2 \quad 1.728389389320815633051329318289$

$5.3 \quad 1.700829345314731775795506112117$

$5.4 \quad 1.673349703841301064667995859280$

$5.5 \quad 1.645957216191491435889025274286$

$5.6 \quad 1.618657845114125283928450808686$

$5.7 \quad 1.591456847794820980913148502729$

$5.8 \quad 1.564358849347146277491869170541$

$5.9 \quad 1.537367908021911303096409328589$

$6.0 \quad 1.510487573170602177382854427622$

$$
\operatorname{dilog}(-x)
$$

$-0.890838090262282605871309428418$ $-0.957405308558781248198010286629$ $-1.022284038302275319986718986228$ $-1.085577956347125075554889582744$ $-1.147380660375570754079976633863$ $-1.207776992369138194073720165256$ $-1.266844145866363447320905517040$ $-1.324652598823732070035912690929$ $-1.381266904633378820281229772061$ $-1.436746366883680946362902023894$ $-1.491145618151680573563637568660$ $-1.544515119048595203885772816970$ $-1.596901590587111272053514639329$ $-1.648348390474691231875756861321$ $-1.698895841995014173048091284015$ $-1.748581522597636561648072986990$ $-1.797440518082914586087229348866$ $-1.845505647276696845334658242627$ $-1.892807661285357115434849326700$ $-1.939375420766708953077271719178$ $-1.985236054115665580574525275757$ $-2.030415099021474096473803050602$ $-2.074936629487449596596800206148$ -2.118823370099811521504826736057 -2.162096799077975095098549495119 $-2.204777241425323351874567636039$ $-2.246883953319760865440055457373$ $-2.288435198731344677614131545051$ -2.32944 8319125246758841438312332 -2.3699397969 98365831985537425350 $-2.409925313903926704546891567960$ -2.449419803537803903885763041577 $-2.488437500390962472800900297322$ -2.52699 1984412570694792946974302 $-2.565096222076555183118930208462$ -2.6027626041994299072948352 33582 $-2.640002980818128376052163241083$ -2.67682 8693402456933013832146069 -2.713250604646956250993800577169 $-2.749279126060808290025587515376$ -2.78492 4243551450236746837890143 $-2.820195541177325162129021900941$ $-2.855102223227345392468201172730$ -2.88965 3134768856704832957071014 -2.92385 6780791902400279072690568 $-2.957721344065167076722392822094$ $-2.991254701807933648399308710288$ $-3.024464441272544185755349106472$ -3.0573578743230689372446768 36844 -3.089942051088031581283046157063 
Bell Laboratories

Murray Hill, New Jersey 07974

1. L. LEWIN, Dilogarithms and Associated Functions, MacDonald, London, 1958.

2. M. ABRAMOWITZ \& I. A. STEGUN, Editors, Handbook of Mathematical Functions, with Formulas, Graphs, and Mathematical Tables, Nat. Bur. Standards Appl. Math. Series \#55, U.S. Government Printing Office, Washington, D. C., 1964.

3. EDWARD S. GINSBERG \& DOROTHY ZABOROWSKY, “Algorithm 490: The dilogarithm function of a real argument," Comm. ACM, v. 18, 1975, pp. 200-202.

4. R. MORRIS, "Remark on Algorithm 490," ACM Trans. Math. Software, v. 2, March 1976, p. 112.

5. JOHN F. HART, E. W. CHENEY, ET AL., Computer Approximations, Wiley, New York, 1968. 